\title{
Design and Channel Constraint Analysis of Ultrafast Multihop All-Optical Networks with Deflection Routing Employing Solitons
}

\author{
Alberto Bononi, Fabrizio Forghieri, and Paul R. Prucnal
}

\begin{abstract}
Regular two-connected multihop transparent optical networks using ultrahigh bit-rate single-wavelength on / off keying are addressed. A novel solution for packet and node architecture is introduced to take full advantage of the recently demonstrated optical samplers and of the electronic processing capability. Channel transmission error arguments show how the size of these nonregenerative networks employing deflection routing is limited for a given optical bit rate. These limits are quantified for the Manhattan Street Network and ShuffleNet employing solitons. An upper bound on network performance in terms of maximum achievable bit rate and throughput for a given packet error rate is evaluated by taking into account the soliton self-frequency shift due to Raman scattering, the jitter due to amplified spontaneous emission noise, the short-range interaction, and their interplay. Results show that if the packet error rate is to be bounded below $10^{-6}$, the node-to-node fiber span cannot exceed a few kilometers for network sizes greater than 64 nodes when the optical bit rate is as high as $100 \mathrm{~Gb} / \mathrm{s}$.
\end{abstract}

\section{INTRODUCTION}

$\mathrm{R}$ EGULAR two-connected mesh topologies for packet switching networks have been proposed for local and metropolitan area networks [1], [2]. Optical fibers, with their low loss and large bandwidth, are a natural choice for the connecting links in high-speed networks of this kind. Traditionally, packets arriving at a node in optical form from the fiber links are converted into electronic form for processing, buffering, and routing, and are then reconverted to optical form for retransmission.

In the all-optical approach, these electrooptic conversions and regenerations are avoided by leaving the packets in optical form as they hop through the nodes toward their destination. All-optical switching makes the intermediate nodes transparent to the packets in transit, so that different bit rates can be accommodated. The channel remains all-optical from source to destination, thereby allowing much higher data rates.

Since neither regeneration of the optical packets nor error control are provided at intermediate nodes, noise

Manuscript received October 19, 1992; revised July 9, 1993.

A. Bononi and P. R. Prucnal are with the Department of Electrical Engineering, Princeton University, Princeton, NJ 08544.

F. Forghieri was with the Department of Electrical Engineering, Princeton University, Princeton, NJ 08544. He is currently with the Crawford Hill Laboratory, AT \& T Bell Laboratories, Holmdel, NJ 07733. IEEE Log Number 9212984. and distortion in the optical fiber channel accumulate as packets propagate so that, for a given optical bit rate, the physical distance from source to destination is constrained if the packet error rate is to be bounded below a given threshold.

Deflection routing [3], [4] may be employed in regular mesh networks, such as the Manhattan Street network (MS [1] and ShuffleNet (SN) [2], to ease the problems arising from all-optical buffering, which is presently difficult to implement at very high bit rates, thereby preventing the use of standard store-and-forward ( $\&$ \&) techniques.

Under deflection routing, repeatedly deflected packets travel long distances before reaching their destination and are thus more likely to be in error at the receiver. In a deflection routing multihop network with equal link lengths, the packet error rate can be obtained by conditioning on the number of hops $n$ taken by a typical packet in the network as

$$
P(e)=\sum_{n=1}^{\infty} P(e / n) P(n) .
$$

The average hop distribution $P(n)$ depends only on network topology, routing, and load, while the conditional probability of packet error $P(e / n)$ only depends on the characteristics of the optical channel, and is a typical point-to-point communication problem, since for a given number of hops and link length the source-destination distance is given.

At ultrahigh bit rates and without regeneration, fiber chromatic dispersion becomes the main system impairment, causing strong intersymbol interference and thus imposing a limit on the maximum propagation distance. To overcome this limitation, solitons may be used because of their dynamic compensation of chromatic dispersion by high-power-induced self-phase modulation (SPM) [5].

. This paper introduces novel solutions for the implementation of the optical nodes in such ultrafast multihop networks, and analyzes the design constraints imposed by the use of soliton packets when deflection routing is employed.

Previous work on multihop networks did not consider the noise characteristics of the optical channel and their impact on network performance. This work concentrates 
on the analysis of the conditional probability $P(e / n)$ for the specific choice of the soliton communication channel. By using the results on $P(n)$ in SN and MS topologies presented in [6], the overall packet error rate is derived from (1) and the impact of the optical channel on the achievable network throughput is found by determining the highest achievable bit rate for a fixed packet error rate, number of nodes, and link-to-link distance. All results are obtained in the case of ideal network synchronization and must then be interpreted as upper bounds on the actual achievable performance.

Section II briefly reviews deflection routing in two-connected regular mesh networks. Section III introduces the proposed all-optical structure of the main blocks of the network nodes. Section IV describes the soliton channel as applied to the ultrafast network environment and derives the conditional probability of packet error $P(e / n)$ for the proposed optical implementation. Section V presents results on packet error probability, maximum optica bit rate and throughput, and link length limitations for MS and SN topologies under deflection routing with no buffers (hot potato) and a single buffer.

\section{Multihop Networks and Deflection Routing}

Slotted fixed-length packet transmission in two-connected multihop networks will be considered, in which each node is basically a cross-bar switch connecting two input links to two output links, capable of transmitting and receiving on both links and of routing packets in transit. When a packet arrives on an input link, its header is read and the best route to its destination, i.e., the best output link, is selected. If both input links have a packet and both packets wish to exit on the same output link, a contention occurs and must be resolved. If buffers are not available, one of the two packets, chosen at random, is deflected on the other output link. This routing strategy, called hot potato [3], can be generalized into the deflection routing algorithm if some buffering is provided [4].

Consider a two-connected multihop network with $N$ nodes at equilibrium. The throughput is defined as the average number of packets inserted/delivered per slot in the network. Let $u$ represent the average link utilization, that is, the average fraction of input links delivering a packet to a node at each clock. The throughput $\lambda$ in bits per second is given by Little's theorem [7] as

$$
\lambda=\frac{2 N}{D} u R,
$$

where $D$ is the average number of hops taken by a packet to reach its destination - the mean of the hop distribution $P(n)$-and $R$ is the bit rate. For shortest path routing, i.e., $\mathrm{S} \& \mathrm{~F}$ with infinite buffers, $D$ is minimized and thus the throughput is maximized. Moreover, $D$ does not depend on the link utilization $u$. If deflection routing is used instead, $D$ becomes an increasing function of $u$, thereby inducing a throughput decrease. Since deflection routing can be implemented all-optically, the loss in efficiency with respect to $S \& F$ can be, in principle, offset by the higher bit rates $R$ allowed by the optical channel [8]. However, for a given network size, that is, number of nodes $N$ and node-to-node separation $l$, the bit rate $R$ is limited by the allowed packet error rate. Finding this limit on $R$ for the soliton channel in $\mathrm{SN}$ and MS topologies will be the object of Section V.

\section{Optical Node Structure}

In the analyzed two-connected networks, the nodes are connected by dedicated fiber links. Figure 1 shows a block diagram of the node structure. Thick lines indicate optical paths and dashed lines indicate electronic controls. There are two local cross-bar switches SW1 and SW2 for packet reception and transmission and a main switch for packet routing. On each input link a small portion of the incoming signal power is stripped off by an optical coupler and sent to the header recognition and routing block. A fiber delay is inserted after the coupler so that incoming packets are sensed before they arrive at the switches, long enough in advance to allow reading the headers, making the routing decisions, and setting the switches. Once a packet's address is recognized to match the node's address, the local switch is set in cross position and the packet is absorbed. At the same time a new packet can be transmitted on the outgoing link. The local switches can be implemented with $\mathrm{LiNbO}_{3}$ waveguides, which are electrically controlled and can have reconfiguration times less than a fraction of nanosecond. Since back-to-back packets must be separated by at least the switch reconfiguration time, even for packets as short as a few nanoseconds, this guard time is a small fraction of the packet duration.

With hot-potato routing the main switch is a simple cross-bar switch like the local switches. Some optical buffers can be added to implement buffered deflection routing. A simple scheme to implement all-optical limited-time buffers using fiber delays has been proposed in [9]. Figure 2 shows the structure of the main switch that implements that scheme for a single-buffer memory. Single-buffer deflection routing already achieves a significant performance improvement over hot potato in terms of throughput and delay. Adding more memory elements does not appreciably improve system performance, while introducing extra losses and complicating the control algorithm [6].

In a slotted network, packets from the two input links must have a fixed length and be aligned at the main switch to be routed in the slot time. The alignment may be implemented with adjustable optical delays [10]. The accuracy with which packets will be aligned depends on the number of adjustable delays.

Amplification is provided at the output links to compensate for the power losses in the fiber and at the node. If one buffer is present, one more switch is needed and thus the amplifier gain must be suitably increased, thereby increasing the noise level.

On/off optical power modulation at a fixed optical wavelength is considered at bit rates far beyond $10 \mathrm{~Gb} / \mathrm{s}$. 


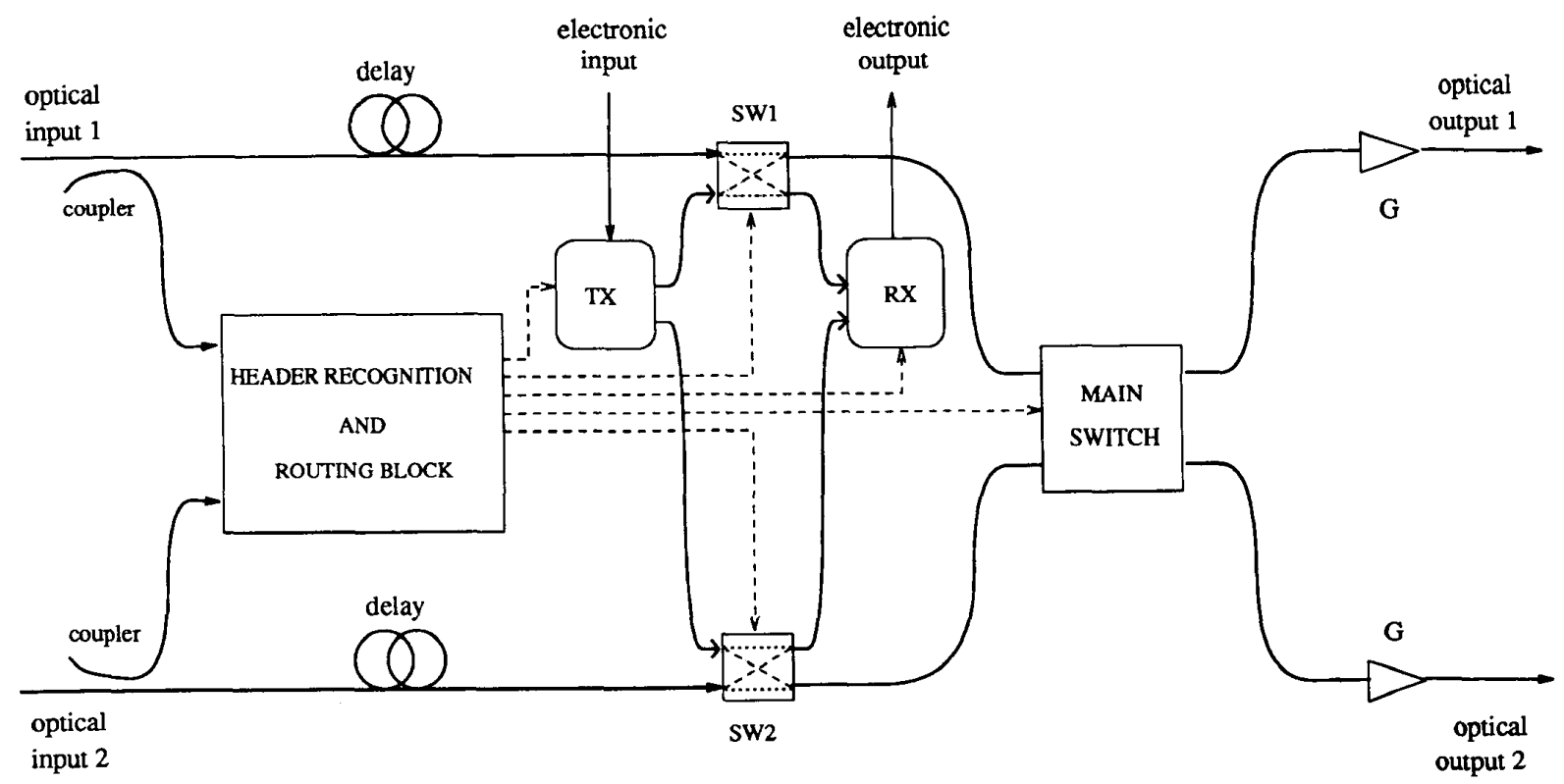

Fig. 1. Node block diagram.

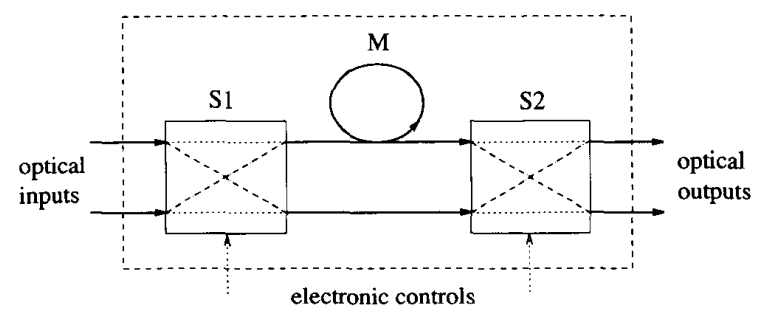

Fig. 2. Main switch with one-packet buffer. $M$ is the delay-line memory. $S 1$ and $S 2$ are exchange-bypass switches.

At the receiver, a packet at such ultrahigh rates must be first decompressed by an all-optical demultiplexer and subsequently detected by a parallel bank of optoelectronic detectors. A all-optical packet demultiplexer can be based on recently demonstrated all-optical sampling gates [11]-[13], in which a high-power sampling optical pulse is synchronized on a specific bit position in the incoming packet, to test whether a bit pulse is present or not. The sampler works thus as an optical AND gate. These gates display some tolerance to jitter between the two pulses, so that a temporal sampling window exists. A major problem is synchronization between the sampling clock and the incoming bit pulse. Practical schemes for synchronization acquisition on a packet-by-packet basis in ultrafast networks are still an open issue and will not be addressed here.

The demultiplexer is a $1 \times k$ optical device that deinterleaves the ultrafast input packet at rate $R$ into $k$ optical streams at lower bit rate $R / k$. With a parallel bank of $k$ optical samplers, packet detection is converted into a parallel detection of extremely short spikes at a repetition rate $R / k$ suitable for electronic detection. The number of output branches $k$ of the demultiplexer can be as high as the number $M$ of bits in the packet, in which case each bit is separately detected in a time as long as the packet duration. However, this number $k$ can be greatly reduced. The following packet construction explains how.

$T X$ block: Figure 3 shows the optical packet generation block in the TX unit. The electronic input is a packet with $h$ header bits and $p$ payload bits, with $p$ an integer multiple of $h, p=n h$. The header is serially fed into a dedicated shift register. The payload, instead, is sequentially separated into $n$ parallel shift registers, as shown in the figure. This initial process can be done at standard low electronic speed. A local optical clock generated by a mode-locked laser produces short optical pulses at a repetition rate $R_{c}=R /(n+1)$, where $R$ is the bit rate in the optical packet. The clock output is split into $n+1$ branches to get one-bit shifted replicas of it, which are fed to an array of $n+1$ electrooptic modulators. Once the electronic packet separation is complete, a strobe signal starts the optical packet modulation. The $n+1$ shift registers feed the parallel bank of modulators at a modulation rate equal to the clock rate $R_{c}$. The modulated optical pulses are recombined into a single stream to yield the optical packet in $h$ clock periods.

Header recognition and routing block: With the previous construction, a spread header packet structure has been obtained [14]. The header, instead of preceding the payload, is spread regularly across the packet in a time-division-multiplexing (TDM) fashion, as seen in Fig. 3. If the interleaving period between header bits is long enough, the header can be extracted by feeding the packet to a 


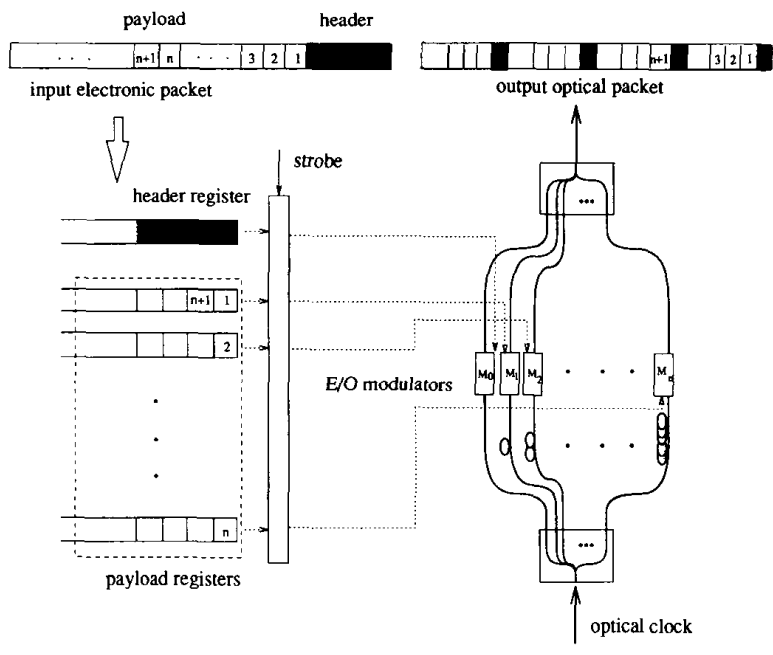

Fig. 3. Optical packet generation block.

single AND gate together with an optical sampling clock at the interleaving rate, and by detecting the output optical signal by an optoelectronic detector. Figure 4 details the header recognition and routing block. A fraction of the power of the incoming packets is stripped off by a coupler to read the header. This fraction must be small to limit the overall packet losses at each node. Amplification is next needed to restore the appropriate pulse power level before all-optical sampling. A properly delayed version of the same optical clock used for packet generation is optically ANDed with the amplified packet replica to extract the header bits, which are then detected by a fast photodiode and stacked into a shift register. Based on the contents of the shift register from each input branch, the desired output for each packet is electronically computed and the switch controls are set. These computations can be pipelined [15] to be performed within a packet duration with the fast algorithms available in regular mesh networks [16].

$R X$ block: Figure 5 shows the scheme of the receiver. The same technique used to extract the header can be used to demultiplex and detect the payload. One-bitshifted versions of the local sampling clock are sent to an array of $n$ optical sampling gates together with the absorbed packet so that demultiplexing by a factor $n$ is achieved. The $n$-sampled sequences at rate $R_{c}$ are separately detected by fast photodiodes and electronically buffered. The output of the $n$ electronic shift registers is finally sequentially scanned to obtain parallel-to-serial conversion and yield the received payload at lower electronic rates. The shift registers should have enough buffers to accommodate a stream of back-to-back optical packets and thus match the network's ultrahigh rates to the lower electronic output rates.

Amplification might need to be supplied to compensate for the splitting losses of both data and clock, according to the power requirements of the all-optical sampling gates employed.

\section{Probability of Error ANalysis}

This section will focus on the impact of the use of ultrashort solitons on network performance. A simple model of the receiver for soliton packets is introduced to evaluate the probability of packet error conditioned on the number of hops $P(e / n)$. An analysis of the noise characteristics of the soliton channel is given in the Appendix.

Very short solitons, of width in the picosecond range, have widely different features from the solitons employed in long-haul communication systems. They have a much higher power, and need to be amplified after much shorter propagation intervals in order not to broaden and disperse like low-power pulses. The main source of noise on very short solitons is time jitter at the receiver. While the amplified spontaneous emission noise (ASE) added by optical amplifiers is still a relevant jitter source [17], another important contribution comes from the soliton self-frequency shift (SSFS) due to Raman scattering, whose effect is inversely proportional to the fourth power of the pulse width [18]. Jitter is also caused by the short-range interaction (SRI) of neighboring solitons [19].

At a fixed bit rate, the pulse width reduction necessary to avoid the short-range interaction strongly enhances the SSFS effect. Distributed amplification will therefore be assumed in the network to compensate for fiber losses, while node losses will be compensated by placing lumped optical amplifiers at each node output. In this way, even ultrashort solitons will propagate without broadening in the span of fiber between nodes, so that they will not get any closer. The initial pulse separation necessary to weaken SRI can thus be decreased, thereby allowing wider soliton pulses and in turn reducing SSFS.

The optical packet demultiplexer/receiver is a bank of parallel optical sampling AND gates. Each AND gate is modeled as a gating window of width $\tau_{w}=w \tau$, where $\tau$ is the soliton full width at half maximum (FWHM) and $w$ will be referred to as the relative window width. The factor $w$ accounts for the sampling time tolerance in the optical sampler. A soliton pulse will come out of the sampling AND gate if its center is inside the corresponding window.

As shown in the Appendix, very short solitons have such a high energy that a strong light pulse will come out of the AND gate, and the optical energy falling inside the following optoelectronic receiver bandwidth is thus high enough to neglect the receiver thermal noise. Moreover, the optical SNR is shown in the Appendix to be high enough to justify the assumption that no errors are made when the soliton is inside the sampling window. Thus errors at the receiver are caused only by jitter of the pulse arrival time in excess of the window width.

Ideal network synchronization is assumed, i.e., each node knows the nominal arrival time of each pulse in the packet, except for the unpredictable jitter accumulated along the optical path. All node operations are synchronous so that packets are transmitted and relayed at the same slot rate $R_{s}=1 / T_{s}$. Define the slot spatial 


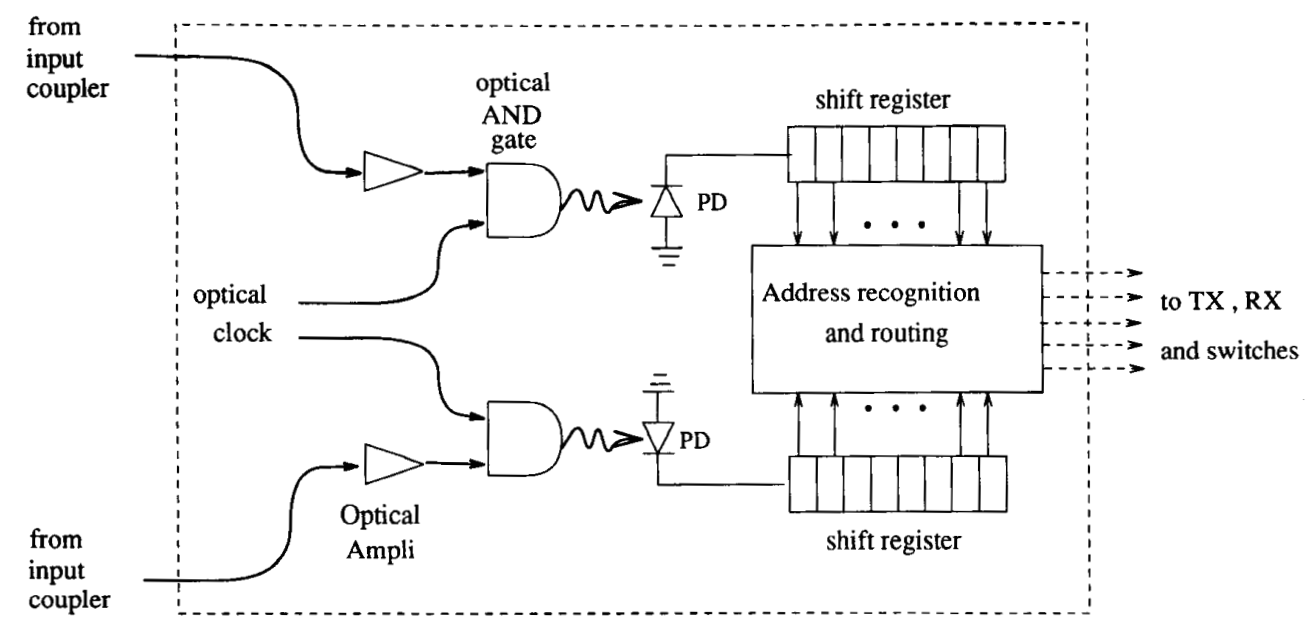

Fig. 4. Header recognition and routing block.

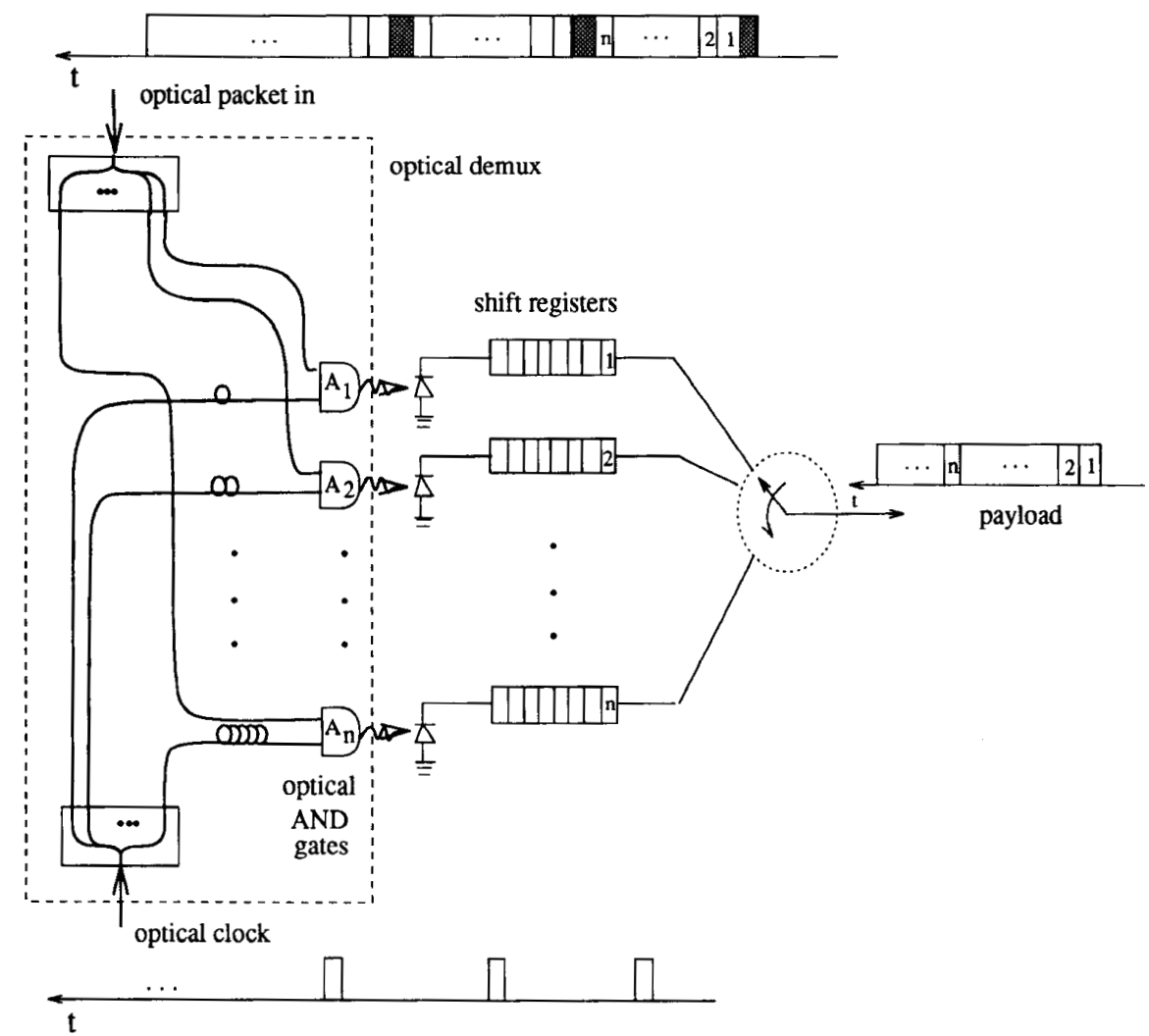

Fig. 5. Receiver block diagram.

length $l_{s}$ as the distance traveled by an ideal slot frame with speed $v_{s}$ in the slot time $T_{s}$. Packets are embedded in this ideal slot frame, in flight from node to node. The speed of each bit in the packet slightly differs from that of the frame $v_{s}$, since it varies during propagation because of
SSFS, ASE noise, and SRI, and this variation induces a jitter of each bit in the packet with respect to its nominal position within the frame. The spatial jitter is detected as a time jitter at the receiver. Focus on a generic bit in the packet and make its nominal arrival time zero. Its time 
jitter $t_{a}$ is the sum of three terms accounting for the above-mentioned effects:

$$
t_{a}=t_{\mathrm{SSFS}}+t_{\mathrm{ASE}}+t_{\mathrm{SRI}} .
$$

Let $t_{L}$ and $t_{U}$ be the start and stop times of the sampling window. Errors only occur when a pulse is outside the window. If " 0 "s and " 1 "s are equally likely, the probability of bit error conditioned on the number of hops $n$ is

$$
P_{b}(e / n)=\frac{1}{2}\left[P\left(t_{a}<t_{L}\right)+P\left(t_{a}>t_{U}\right)\right] .
$$

The single contributions to $t_{a}$ will now be outlined.

SSFS: As shown in the Appendix, the Raman shift $t_{\text {SSFS }}$ is the same for all bits in a packet and is a deterministic function of the propagation distance $z=n l$, where $n$ is the number of hops taken by the packet in its travel and $l$ is the link length. Randomness in $t_{\text {SSFS }}$ arises when deflection routing is used, since the received packets have hopped a random number of times when they arrive at their destination.

If the slot frame is chosen to propagate with speed slightly lower than the soliton's initial group velocity, the Raman shift $t_{\mathrm{SSFS}}$ is from (A.4),

$$
t_{\mathrm{SSFS}}=T_{R} n^{2}-T_{D} n,
$$

where $T_{R}$ is the Raman shift in one hop of length $l$ with respect to a frame moving with the soliton initial group velocity (the soliton retarded frame), and the second term on the right-hand side (RHS) represents the drift of the slot frame with respect to the soliton retarded frame.

ASE: The ASE-induced shift $t_{\mathrm{ASE}}$ is a zero mean Gaussian random variable, independent bit by bit, whose variance $\sigma_{\mathrm{ASE}}^{2}$ is given in (A.5).

SRI: Assuming that the solitons in the packet have equal amplitude and phase, as discussed in the Appendix, the short-range interaction of neighboring solitons is a deterministic attractive force. The shift $t_{\mathrm{SRI}}$ is a deterministic function of the hop number $n$ and of the pattern of pulses around the bit under test. Interaction forces between nonneighboring pulses are negligible. Therefore it is only necessary to consider the bit preceding and the one following the bit under test to determine the drag. The random variable $X$ will indicate whether no forces are present on the test pulse ( $X=0$ for the 010 pattern), if it is dragged to the left $(X=-1$ for 110$)$, or to the right ( $X=1$ for 011 ). In the unspecified case 111, two more side bits must be considered to determine the drag. By assuing that all bit configurations are equally likely, it is easily found that $X$ assumes values $-1,0,1$ with equal probability. Within the same set of configurations for which $X=1$ (or $X=-1$ ) the attraction forces do not have the same intensity. However, a worst-case situation can be considered in which the attraction has maximum strength, as is the case when only two neighboring pulses are interacting. In this case the absolute value of $t_{\mathrm{SRI}}$ can be found from (A.6):

$$
\left|t_{\mathrm{SRI}_{\max }}\right|=\frac{t_{s}}{2}\left\{\ln 2-\ln \left[1+\cos \left(2 \pi \frac{n l}{Z_{0}} e^{-T / 2 t_{s}}\right)\right]\right\},
$$

where $T$ is the bit time, $t_{s}$ is the soliton width parameter defined in (A.2), and $Z_{0}$ is the soliton characteristic length given in (A.3). It is thus a deterministic function of the hop number $n$, and it strongly depends on the normalized initial pulse separation $T / t_{s}$.

A simple worst-case approximation is obtained by assuming that all bits are independently affected in the packet and by modeling the random variable $t_{\mathrm{SRI}}$ as

$$
t_{\mathrm{SRI}}=X\left|t_{\mathrm{SRI}_{\max }}\right| .
$$

Edge effects can be neglected in long packets, as in the ATM standard of about 500 bits.

ASE-SRI interplay: The ASE jitter $t_{\mathrm{ASE}}$ may cause two neighobring pulses to get closer together, thereby accelerating the short-range interaction. This interference has already been studied in [20]. For the case of two in-phase, equal amplitude solitons in a 110 or 011 pattern, where the center bit is the bit under test, it is shown there that, for propagation distance

$$
z=n l \leq 0.36 Z_{0} e^{T / 2 t_{s}},
$$

the mutual interaction between ASE and SRI is small so that, upon conditioning on $X$, the random variable $\left(t_{\mathrm{ASE}}\right.$ $\left.+t_{\mathrm{SRI}}\right)$ may be considered Gaussian with mean $X \mid t_{\mathrm{SRI}}$ and variance $\hat{\sigma}=F(z) \sigma_{\mathrm{ASE}}$, where the function $F(z)$ (given in [20], Fig. 4) increases approximately exponentially with propagation distance $z$. Including also the 010 pattern where no interactions are present, the variance of $\left(t_{\mathrm{ASE}}+t_{\mathrm{SRI}}\right)$ is

$$
\sigma=\left(1-X^{2}\right) \sigma_{\mathrm{ASE}}+X^{2} \hat{\sigma} .
$$

For a fixed bit time $T,\left|t_{\mathrm{SRI}_{\max }}\right|$ is negligible if the pulses are initially well separated, i.e., $T / t_{s}$ is very large, but the Raman shift is greatly enhanced since $t_{s}$ becomes very small. On the other hand, if $T / t_{s}$ is small, SRI would cause the collapse of neighboring pulses within short distances. A conservative criterion to select $T / t_{s}$ has been used so that approximation (9) is satisfied down to a target packet error probability of $10^{-12}$. To achieve this, a maximum number of hops of interest in the network $n_{\max }$ is found so that

$$
\sum_{n=n_{\max }+1}^{\infty} P(n) \leq 10^{-12} .
$$

The value of $T / t_{s}$ is selected so that, at a distance $z_{\max }=n_{\max } l$, relation (8) holds with equality, which corresponds to a maximum SRI shift $\left|t_{\mathrm{SRI}_{\max }}\right| \simeq \tau / 2$.

The conditional bit error probability $P_{b}(e / n)$ can now be obtained by putting the previous results together. By conditioning on the random variable $X$ and using rela- 

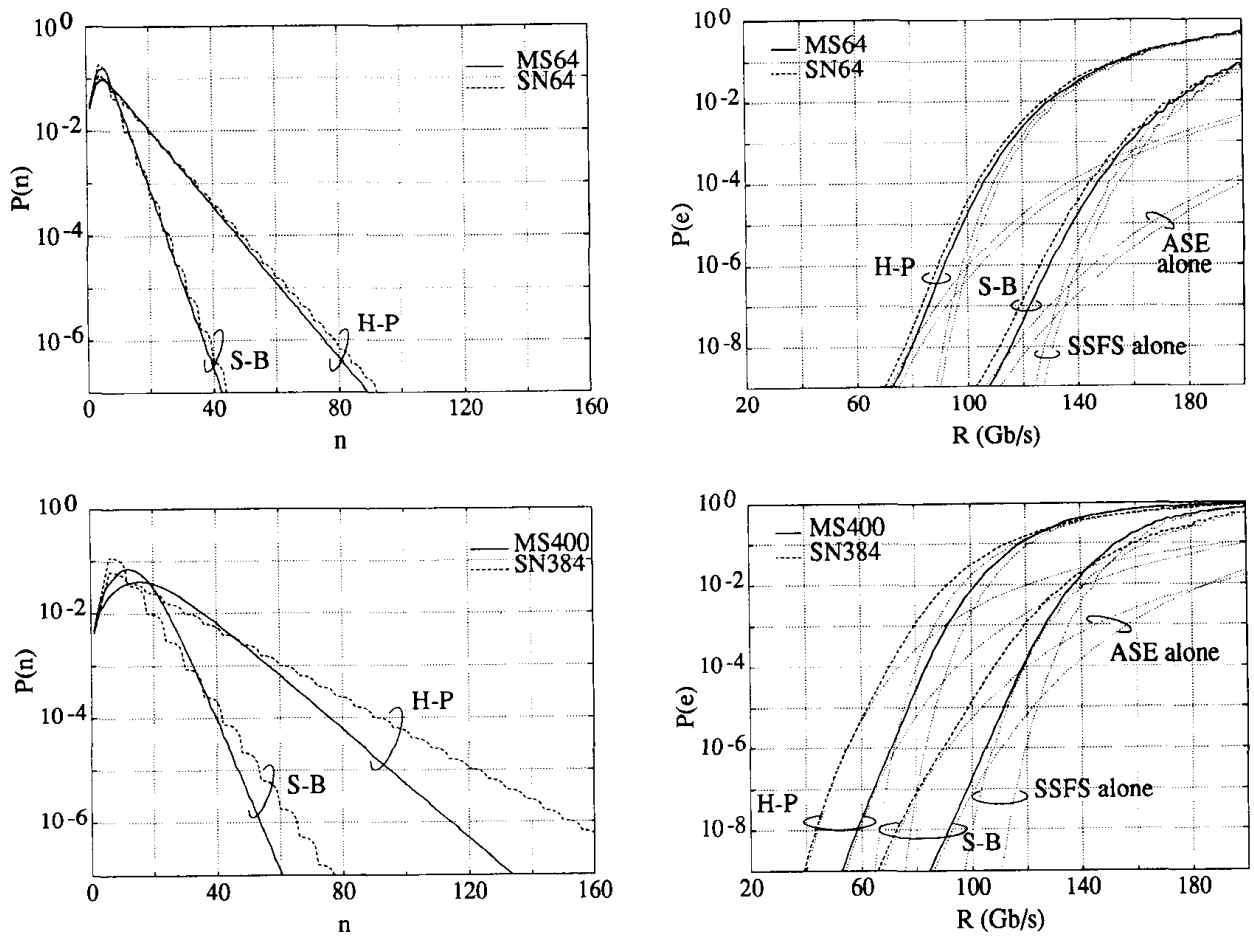

(a)

(b)

Fig. 6. (a) Hop distribution $P(n)$ for MS and SN with hot-potato routing (H-P) and single-buffer deflection routing (S-B) at full load $(g=1)$. (b) Corresponding packet error probability $P(e)$ for 1-km link length. The contributions of ASE and SSFS when they are the only source of jitter are also shown in dotted lines.

tions (5) and (7), the shift $t_{a}$ in (3) becomes a Gaussian random variable with mean $E t_{a}(X, n)=T_{R} n^{2}-T_{D} n+$ $X\left|t_{\text {SRI }}\right|$ and variance $\sigma(X, n)$ given in (9). Hence, by the law of total probability, relation (4) becomes

$$
\begin{aligned}
P_{b}(e / n)=\frac{1}{6} \sum_{X}\left[Q\left(\frac{t_{U}-E t_{a}(X, n)}{\sigma(X, n)}\right)\right. & \\
& \left.+Q\left(\frac{E t_{a}(X, n)-t_{L}}{\sigma(X, n)}\right)\right],
\end{aligned}
$$

where the summation runs over the possible values of $X$ and $Q(x)=(1 / \sqrt{2 \pi}) \int_{x}^{\infty} \exp \left(-t^{2} / 2\right) d t$.

Each bit is assumed to be independently affected in the packet so that the packet error rate is finally, from (1),

$$
\begin{aligned}
P(e) & =\sum_{n}\left[1-\left[1-P_{b}(e / n)\right]^{M}\right] P(n) \\
& \simeq M \sum_{n} P_{b}(e / n) P(n),
\end{aligned}
$$

where $M$ is the number of bits in the packet, and the RHS approximation holds for low values of $P(e)$ and shows its linear dependence on the packet size $M$.

\section{RESULTS}

Hop distribution curves for MS and SN using both single-buffer deflection routing and hot-potato routing have been obtained as described in [6] for 64-node network size (MS64 and SN64) and 400-node network size
(MS400 and SN384), and are presented in Fig. 6(a) at full load. The essential feature is that $\mathrm{SN}$ has lower mean $D$, but higher tail probability than MS.

Figure 6(b) shows the corresponding packet error rate curves, obtained from (11), plotted versus the optical bit rate $R$. The results were obtained for $1-\mathrm{km}$ link length, fiber loss $0.25 \mathrm{~dB} / \mathrm{km}$, fiber dispersion parameter $D=1$ $\mathrm{ps} / \mathrm{nm} / \mathrm{km}$, ATM packet size $M=424$ bits $/$ packet, relative window $w=4$, and node amplifier gain per channel $G=10 \mathrm{~dB}$ for hot-potato and $G=15 \mathrm{~dB}$ for single-buffer deflection routing. The pulse separation factor $T / \tau$, selected as explained in the lines below (10), ranged from 4 (the window size) to about 10 as the bit rate was increased from 20 to $200 \mathrm{~Gb} / \mathrm{s}$. Error probability curves when the only source of jitter is, respectively, ASE alone or SSFS alone are also provided in dotted lines. As expected, the SSFS jitter dominates at high values of $R$ (very short bit pulses), while the ASE jitter dominates at lower bit rates.

Errors are due to packets that have hopped a high number of times. The $P(e / n)$ curves are steplike functions, rising abruptly from 0 to 1 as $n$ increases, as shown in Fig. 7. From (1), the value of $P(e)$ is roughly the integral of the portion of the hop distribution tail that is encompassed by $P(e / n)$. The error probability in $\mathrm{SN}$ is thus higher than in MS because of the tail behavior of $P(n)$. The use of deflection routing with a single fiber delay line buffer drastically lowers the hop distribution 

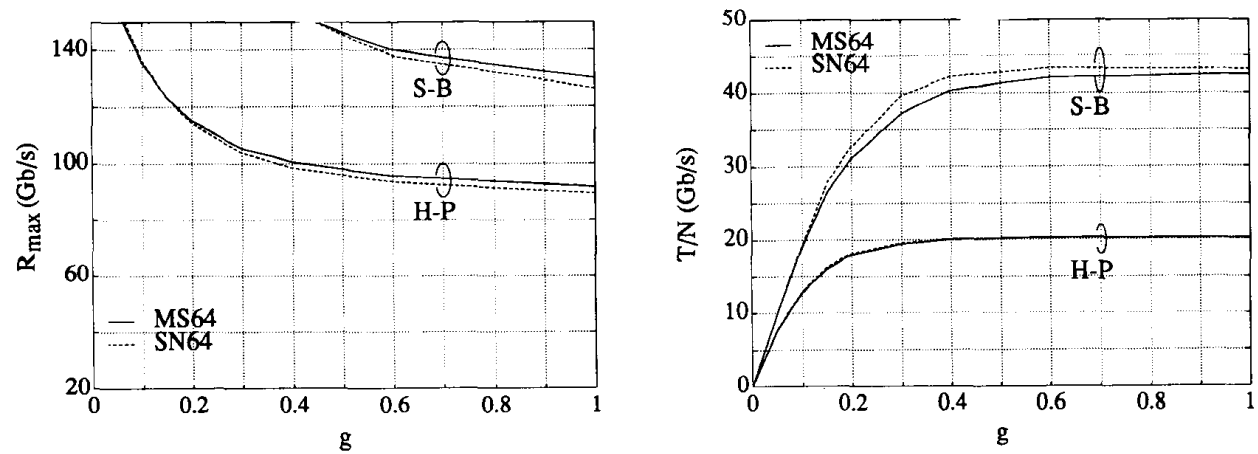

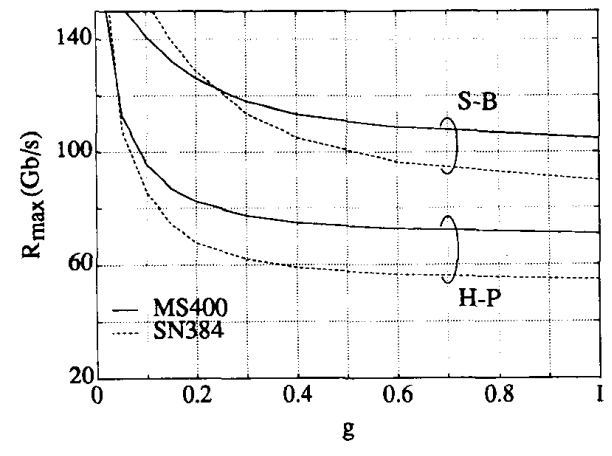

(a)

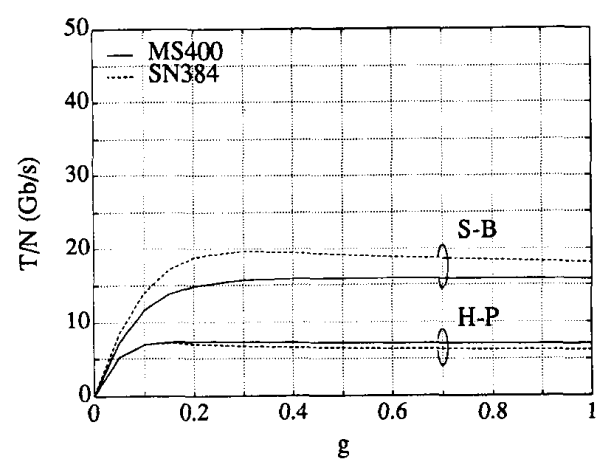

(b)

Fig. 8. (a) Maximum optical bit rate $R_{\max }$ to achieve $P(e) \leq 10^{-6}$ and (b) corresponding throughput per node $T / N$ vs. offered load $\mathrm{g}$ in MS and SN with 1-km link length.

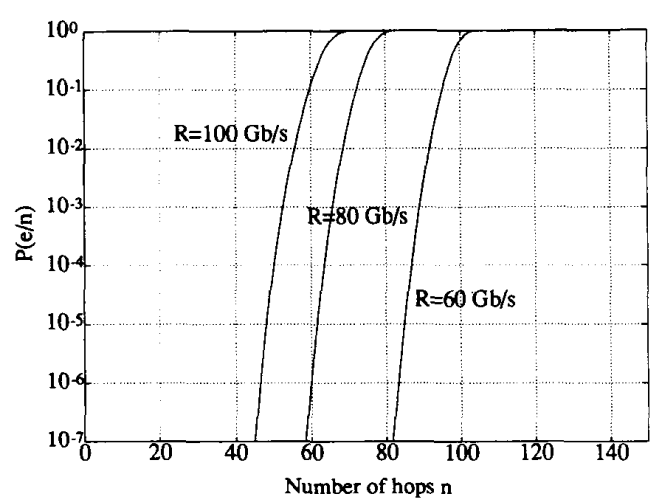

Fig. 7. Conditional packet error probablity $P(e / n)$ versus number of hops $n$ for link length $1 \mathrm{~km}$, amplifier gain $15.25 \mathrm{~dB}$, and for bit rate $R=60,80$, and $100 \mathrm{~Gb} / \mathrm{s}$.

tail, so that much higher bit rates can be obtained for the same packet error rate with respect to the case of no buffers (hot potato).

A comment on the possible use of filtering techniques to improve performance is in order. The sliding-guiding filter technique recently proposed by Mollanauer et al. [21] is not suitable for a multihop network environment in which the path from source to destination is dynamically allocated. Also the temporal-filtering technique of Nakazawa et al. is not suited to these ultrafast networks, because a direct modulation of the optical soliton packet at these ultrafast bit rates would be necessary. As for the center-guiding filters [23], [24], it is shown in [23] that the advantage of filtering can be enjoyed only after a propagation distance $L_{0}=K l[(15)]$, where $l$ is the spacing between amplifiers and $K$ is a proportionality constant. Assuming as in [25] amplification stage filters of bandwidth 10 times the soliton bandwidth, one gets $K \cong 90$. Now, as shown in Fig. 7, packets that are in error at rates higher than $80 \mathrm{~Gb} / \mathrm{s}$ are those that have hopped more than approximately 70 times, not enough to enjoy the positive filter action. This implies that the $P(e)$ curves in Fig. 6(b) will be lowered in the low-bit-rate range, below $60 \mathrm{~Gb} / \mathrm{s}$, but nothing will substantially change for the 64-node networks, while in the 400 -node networks the most significant curves, those referring to single-buffer, will not be substantially affected.

Sensitivity plots at $P(e)=10^{-6}$ have been extracted from the packet error probability curves obtained for various values of the offered load $g$ and of the link length $l$.

Figure 8 shows the maximum bit rate $R_{\max }$ for which the error probability is below $10^{-6}$ [curve (a)] and the corresponding throughput per node obtained from (2) [curve (b)] versus the offered load $g$, which is defined as the probability that a packet is ready for transmission at each node in each temporal slot. In (2) both the link utilization $u$ and the average number of hops $D$ are increasing functions of the offered load $g$. For fixed $R$, the throughput is higher in SN since $D$ is lower than in 

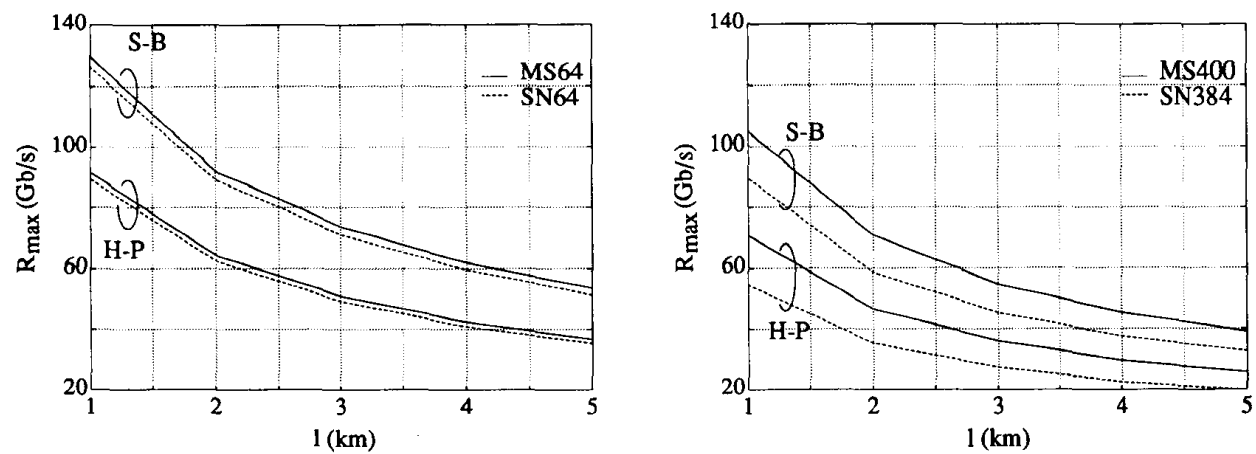

Fig. 9. Maximum optical bit rate $R_{\max }$ to achieve $P(e) \leq 10^{-6}$ vs. link length, at full load and with $w=4$.

MS for every value of $g$ [6]. However, since under hot potato the tail of the hop probability in MS is much lower than in $\mathrm{SN}$, much higher bit rates $R$ are possible in MS for a given value of $P(e)$. This offsets the initial disadvantage of MS, so that its throughput is higher than in SN under hot potato. An important result is that whenever the hop probability tail is substantially decreased, as happens when a single optical buffer is used with deflection routing, SN regains its lead and displays a higher throughput. This is more evident in the 400 node size, where the presence of a maximum at $g=0.3$ can be clearly observed. SN should then be preferred to MS in this ultrafast soliton channel whenever at least one optical buffer is provided. Some form of flow control might be provided in $\mathrm{SN}$ to maximize the throughput. The same figures also show that providing one optical buffer more than doubles the maximum achievable throughput. This results from the combined reduction of the hop distribution mean $D$ and increase of the maximum bit rate $R$, both due to the reduced number of deflections in the network.

Figure 9 shows $R_{\max }$ as the link length varies from 1 to $5 \mathrm{~km}$, at full load and for $w=4$. It can be noted that adding one optical buffer has almost the same effect as halving the link length. In the 400 -node size and with one optical buffer, from about $100 \mathrm{~Gb} / \mathrm{s}$ at $l=1 \mathrm{~km}$ the optical bit rate quickly decreases to about $40 \mathrm{~Gb} / \mathrm{s}$ at $l=5 \mathrm{~km}$, while it sinks below $20 \mathrm{~Gb} / \mathrm{s}$ with no buffers. Numerical values are better for the smaller 64-node size, but the general results suggest that ultrafast soliton alloptical networks without regeneration at bit rates around $100 \mathrm{~Gb} / \mathrm{s}$ could only be conceived for local or metropoli$\tan$ area networks, with little more than $1-\mathrm{km}$ fiber span from node to node in networks with more than 64 nodes, and using at least one optical buffer.

\section{Conclusions}

Recently demonstrated all-optical sampling techniques for TDM demultiplexing can be applied to header recognition and packet detection in all-optical packet switching networks. Spreading of the header over the packet in a TDM fashion allows both header recognition with only one optical sampling gate and minimization of the number of sampling gates in the receiver. The proposed schemes emphasize the use of electronic processing where an all-optical counterpart is not available at present.

An analysis of the packet error rate in the nonregenerative optical soliton channel at ultrahigh bit rates provides bounds on the achievable network size and maximum throughput. The packet error probability computation ignores the separate processing of the packet's header at the intermediate nodes. Ideal slot synchronization is also assumed at all nodes. The results of this simplified model show that ultrafast soliton all-optical SN or MS networks without regeneration at bit rates of about $100 \mathrm{~Gb} / \mathrm{s}$ could only be conceived for local or metropolitan area networks, with little more than 1-km fiber span from node to node for network sizes exceeding 64 nodes. If wider networks are to be implemented, regeneration of the optical packet must be provided.

Another important result is that the use of a single fiber delay optical buffer has a much greater impact on network performance than expected from the classical network approach which neglects errors in the channel [6]. In fact, the throughput is more than doubled with respect to the case where no buffers are used.

\section{APPENDIX}

The nonlinear Schrödinger equation (NLSE) describing, in the retarded frame, the propagation of the envelope $u(z, t)$ of the electric field in a lossless single-mode fiber is [5]

$$
i \frac{\partial u}{\partial z}=\frac{\beta^{\prime \prime}}{2} \frac{\partial^{2} u}{\partial t^{2}}-\sigma u|u|^{2},
$$

where $z$ is the propagation distance, $t$ is the retarded time, and $\beta^{\prime \prime}$ is the group velocity dispersion (GVD) parameter, $\sigma$ is a nonlinear parameter defined by $\sigma \triangleq$ $n_{2} \omega_{0} / c A_{\text {eff }}$, where $n_{2}$ is the Kerr coefficient, $\omega_{0}$ is the pulse carrier angular frequency, $c$ the light speed, and $A_{\text {eff }}$ is the effective area of the light mode profile in the fiber. $\beta^{\prime \prime}$ is related to the chromatic dispersion parameter $D$ by $\beta^{\prime \prime}=-\left(2 \pi c / \omega_{0}^{2}\right) D$.

The fundamental soliton is a solution of (A.1) of the form

$$
u(z, t)=\frac{1}{t_{s}} \sqrt{\frac{\left|\beta^{\prime \prime}\right|}{\sigma}} \exp \left(-\frac{i\left|\beta^{\prime \prime}\right| z}{2 t_{s}^{2}}\right) \operatorname{sech}\left(\frac{t}{t_{s}}\right) .
$$


The soliton FWHM $\tau$ is related to the width parameter $t_{s}$ by $\tau=2 \cosh ^{-1} \sqrt{2} t_{s} \approx 1.763 t_{s}$.

In the retarded frame, due to the interplay between chromatic dispersion and the Kerr effect, the soliton propagates unchanged except for a small phase rotation. The length over which the phase rotates by an angle of $\pi / 4$ is the so-called soliton period

$$
Z_{0}=\frac{\pi}{2} \frac{t_{s}^{2}}{\left|\beta^{\prime \prime}\right|} \propto \frac{\tau^{2}}{D} .
$$

This length is a characteristic scale for the soliton evolution, and decreases with the square of the pulsewidth.

Equation (A.2) shows that the phase of the soliton is constant over the whole pulse, being a function of $z$ only. Since optical switching is a phase-dependent process, switching of the whole soliton can be obtained with high efficiency.

The energy of the soliton pulse given in (A.2) is $E=$ $2\left|\beta^{\prime \prime}\right| / \sigma t_{s} \propto D / \tau$. If $R=1 / T$ is the bit rate and $\theta=$ $T / \tau$ is the ratio between bit time and FWHM of the soliton, and assuming that " 0 "s and " 1 "s are equally likely, the average optical power in the fiber is $P=$ $E /(2 \theta \tau) \propto D / \tau^{2}$. At $\lambda_{0}=1.55 \mu \mathrm{m}$ and $R=100 \mathrm{~Gb} / \mathrm{s}$, using $n_{2}=3.18 \times 10^{-16} \mathrm{~cm}^{2} / \mathrm{W}, A_{\text {eff }}=50 \mu \mathrm{m}^{2}, D=1$ $\mathrm{ps} / \mathrm{nm} / \mathrm{km}$, and $\theta=10$, the average power can be found to be $P \approx 20 \mathrm{dBm}$. Therefore, optical amplifiers with output saturation power exceeding $100 \mathrm{~mW}$ are needed at the nodes. Although very high, this value lies in the range of achievability of erbium-doped fiber amplifiers [26].

The number of photons per bit time $T$ added by the amplified spontaneous emission in each amplifier is $N_{\text {amp }}$ $=\beta_{e}(G-1) B_{\mathrm{opt}} / R$, where $\beta_{e}$ is the excess noise factor, $G$ is the linear amplifier gain, and $B_{\text {opt }}$ is the amplifier optical bandwidth. With the above values, the number of photons per soliton pulse is of the order of $10^{7}$, while $N_{\text {amp }}$ is about 500 for $\beta_{e}=1.5, G=10$, and $B_{\text {opt }}=4$ THz. Even cascading 100 of these amplifiers, the optical SNR is still above $25 \mathrm{~dB}$, which guarantees practically error-free detection in the absence of jitter.

\section{A. Soliton Self-Frequency Shift}

Stimulated Raman scattering is a nonlinear effect in which higher-frequency components of light propagating through the fiber act as a pump for lower-frequency components, thereby transferring energy from high to low frequencies [27]. For very short soliton pulses this effect has been shown [18] to produce a continuous downshift of the pulse-carrier frequency with rate $d \nu_{0} / d z=2.9 \times 10^{-3}$ $D / \tau^{4}(\mathrm{THz} / \mathrm{km})$, where $D$ is in picoseconds per nanometer per kilometer and $\tau$ in picoseconds. This frequency shift is linear with the propagation distance $z$ and causes a linear increase in the pulse speed through GVD, hence a constant acceleration. In the retarded frame moving with the group velocity of the initial carrier frequency $\omega_{0}=2 \pi \nu_{0}$ of the soliton, the time shift $t_{\mathrm{SSFs}}(z)$ of the soliton peak position is given in picoseconds by $t_{\mathrm{SSFS}}(z)=$ $6.8 \times 10^{-3} \beta^{\prime \prime} z^{2} / \tau^{4}$, where $\beta^{\prime \prime}$ is in square picoseconds per kilometer and the propagation distance $z$ in kilometers [28].
More generally, in a frame moving with the group velocity relative to the frequency $\omega=\omega_{0}-\Delta \omega$, the time shift $t_{R}^{\Delta \omega}(z)$ of the soliton peak is

$$
t_{R}^{\Delta \omega}(z)=6.8 \times 10^{-3} \frac{\beta^{\prime \prime 2}}{\tau^{4}} z^{2}-2 \pi \beta^{\prime \prime} \Delta \omega z .
$$

Fluctuations of the soliton energy $\Delta E / E=\epsilon$ cause fluctuations of the soliton pulse width $\Delta \tau / \tau=\epsilon$ and thus of the time shift $\Delta t_{\mathrm{SSFS}} / t_{\mathrm{SSFS}}=(1-4 \epsilon)$. If the photon number is Gaussian distributed with variance equal to the mean, with $\tau$ of the order of $1 \mathrm{ps}$ and with the fiber parameters used above, the energy fluctuation $\epsilon$ within 6 times the standard deviation is around $2 \times 10^{-3}$, and the contribution $4 \epsilon$ to the time shift $t_{\mathrm{SSFS}}(z)$ can be safely neglected.

\section{B. Gordon-Haus Effect}

Optical amplification is needed in the network to compensate for the losses at the nodes and in the fiber. It is assumed that an optical amplifier with gain $G_{c}$ is placed at every node on each output link, and that the node-to-node fiber losses are compensated by using distributed amplification with overall gain $G_{d}=\alpha l$, where $\alpha$ is the power loss coefficient and $l$ is the link length.

The ASE noise is a Gaussian random process which perturbs the central frequency of the soliton in a random way [17]. It can be considered a white process since its bandwidth is much larger than the signal bandwidth, so that each soliton in the bit stream is independently affected. The Gaussian frequency shift $\delta \nu$ induced by ASE causes a change in speed $\delta v_{g}$ through GVD, hence a Gaussian time shift $\delta t$ in the retarded frame which is linear in $z$.

In the calculations, the ASE noise added by the distributed amplifier on a link will be treated as if it were added at the upstream node, by supposing that the node amplifiers have gain $G=G_{c} G_{d}$. This is a good approximation, since for link lengths of interest it is $G_{c} \gg G_{d}$. The result of this approximation is to slightly overestimate the effect of ASE.

If the path taken from source to destination is composed of $n$ hops of length $l$ each, and amplification is provided at the beginning of each hop, then the total time shift after $n$ hops is $t_{\mathrm{ASE}}=\sum_{i=0}^{n-1} \delta t_{i} \propto \sum_{i=0}^{n-1} \delta \nu_{i}(n-i) l$, where $\delta \nu_{i} \mathrm{~s}$ are zero-mean i.i.d. Gaussian random variables. $t_{0}$ itself is thus a zero-mean Gaussian random variable, with variance [17]

$$
\sigma_{\mathrm{ASE}}^{2}=\beta_{e} \frac{G-1}{3} \frac{D n_{2} h}{A_{\text {eff }}} \frac{f(n)}{t_{s}} l^{2},
$$

where $h$ is Planck's constant and $f(n)=\sum_{i=0}^{n-1}(n-i)^{2}=$ $n^{3} / 3+n^{2} / 2+n / 6$.

\section{Short-Range Interaction}

As shown by Gordon in [19], neighboring solitons exert forces on each other, while propagating in the optical fiber, because of their nonlinear nature. These forces decrease exponentially with pulse separation and are attractive or repulsive depending on the pulses' relative phase. In this work it is assumed that solitons are gener- 
ated by a mode-locked laser and are subsequently split into $n+1$ pulses which are separately modulated and then recombined to form the packet. Even the pulses that originate from the same soliton will have random phases after recombination, since the path difference between the various branches is much longer than the optical wavelength and will never contain exactly an integer number of optical carrier cycles. However, as a worst case, all the solitons in a packet will be considered to have the same phase, since this corresponds to maximum attractive force. The time separation $\Delta T(z)$ of two equal-phase solitons whose initial separation is $\Delta T(0)=T$ is given as a function of the propagation variable $z$ by [5]

$$
\Delta T(z)=T-t_{s} \ln \left[\frac{2}{1+\cos (\pi z / \zeta)}\right],
$$

where $\zeta=\left(Z_{0} / 2\right) \exp \left(T / 2 t_{s}\right)$ is the distance at which the two solitons collapse.

\section{ACKNOWLEDGMENT}

The authors are grateful to Michel Chbat and Pat Iannone for many useful discussions.

\section{REFERENCES}

[1] N. F. Maxemchuk, "The Manhattan Street network," in Proc. GLOBECOM '85, pp. 255-261.

[2] A. S. Acampora, M. J. Karol, and M. G. Hluchyj, "Terabit lightwave networks: The multihop approach," $A T \& T T e c h$. J., vol, 66, pp. $21-34$, Nov./Dec. 87.

[3] P. Baran, "On distributed communications networks," IEEE Trans. Commun. Syst., vol. COM-12, pp. 1-9, 1964.

[4] N. F. Maxemchuk, "Comparison of deflection and store-and-forward techniques in the Manhattan Street and Shuffle-Exchang networks," Proc. IEEE INFOCOM '89, Apr. 1989, pp. 800-809.

[5] G. P. Agrawal, Nonlinear Fiber Optics. New York: Academic Press, 1989

[6] F. Forghieri, A. Bononi, and P. R. Prucnal, "Analysis and comparison of hot-potato and single buffer deflection routing in very high bit rate optical mesh networks," IEEE Trans. Commun., to be published.

[7] D. Bertsekas, R. Gallager, Data Networks. Englewood Cliffs, NJ: Prentice-Hall, 1987.

[8] A. S. Acampora and A. Shah, "Multihop lightwave networks: A comparison of store-and-forward and hot-potato routing," IEEE Trans. Commun., vol. 40, pp. 1082-1090, June 1992.

[9] I. Chlamtac and A. Fumagalli, "An all-optical switch architecture for manhattan networks," IEEE J. Select. Areas Commun., vol. 11, pp. 550-559, May 1993.

[10] P. R. Prucnal, "Optically processed self-routing, synchronization, and contention resolution for 1-D and 2-D photonic switching architectures," IEEE J. Quantum Electron., vol. 29, pp. 600-612, Feb. 1993.

[11] M. N. Islam, "Ultrafast al-optical logic gates based on soliton trapping in fibers," Opt. Lett., vol. 14, pp. 1257-1259, Nov. 1989.

[12] P. A. Andrekson, N. A. Olsson, M. Haner, J. R. Simpson, T Tanbun-ek, R. A. Logan, D. Coblents, H. M. Presby, and K. W. Wecht, " $32 \mathrm{~Gb} / \mathrm{s}$ optical soliton data transmission over $90 \mathrm{~km}$," IEEE Photon. Technol. Lett., vol. 4, pp. 76-79, Jan. 1992.

[13] P. A. Andrekson, N. A. Olsson, J. R. Simpson, D. J. Digiovanni, P. A. Morton, T. Tanbun-Ek, R. A. Logan, and K. W. Wecht, "64 $\mathrm{Gb} / \mathrm{s}$ all-optical demultiplexing with the nonlinear optical-loop mirror," IEEE Photon. Technol. Lett., vol. 4, pp. 644-647, June 1992.

[14] F. Forghieri, A. Bononi, and P. R. Prucnal, "Novel packet architecture for all-optical ultrafast packet switching networks," Electron. Lett., vol. 28, pp. 2289-2291, Dec. 1992.

[15] P. R. Prucnal and P. A. Perrier, "Optically-processed routing for fast packet switching," IEEE LCS Mag., vol. 1, pp. 54-67, May 1990.

[16] N. F. Maxemchuk: "Routing in the Manhattan Street network," IEEE Trans. Commun., vol. COM-35, pp. 503-512, May 1987.
[17] J. P. Gordon and H. A. Haus, "Random walk of coherently amplified solitons in optical fiber transmission," Opt. Lett., vol. 11, pp. $665-667$, Oct. 1986 .

[18] J. P. Gordon, "Theory of the soliton self-frequency shift," Opt Lett., vol. 11, pp. 662-664, Oct. 1986.

[19] __ "Interaction forces among solitons in optical fibers," Opt. Lett., vol. 8, pp. 596-598, Nov. 1983.

[20] T. Georges, "Amplifier noise jitter of two interacting solitons," Opt. Commun. Mag. pp. 195-201, 1991.

[21] L. F. Mollenauer, J. P. Gordon, and S. G. Evangelides, "The sliding-frequency guiding filter: An improved form of soliton jitter control," Opt. Lett., vol. 17, pp. 1575-1577, Nov. 15, 1992.

[22] M. Nakazawa, E. Yamada, H. Kubota, and K. Suzuki, "10 Gbit/s soliton data transmission over one million kilometres," Electron. Lett., vol. 27, pp. 1270-1273, 1991.

[23] A. Mecozzi, J. D. Moores, H. A. Haus, and Y. Lai, "Soliton transmission control," Opt. Lett., vol. 16, pp. 1841-1843, Dec. 1991.

[24] Y. Kodama and A. Hasegawa, "Generation of asymptotically stable optical solitons and suppression of the Gordon-Haus effect," Opt. Lett.; vol. 17, pp. 31-33, Jan. 1, 1992.

[25] D. Marcuse, "Simulations to demonstrate reduction of the Gordon-Haus effect," Opt. Lett., vol. 17, pp. 34-36, Jan. 1, 1992.

[26] S. G. Grubb, W. F. Humer, R. S. Cannon, T. H. Windhorn, S. W Vendetta, K. L. Sweeney, P. A. Leilabady, W. L. Barnes, K. P. Jedrzejewski, and J. E. Townsend, " $+21 \mathrm{dBm}$ erbium power amplifier pumped by a diode-pumped Nd:YAG laser," IEEE Photon. Technol. Lett., vol. 4, pp. 553-555, June 1992.

[27] R. H. Stolen, "Nonlinearity in fiber transmission," Proc. IEEE, vol. 68 , pp. $1232-1236$, Oct. 1980 .

[28] D. Wood, "Constraints on the bit rates in direct detection optical communication systems using linear or soliton pulses," J. Lightwave Technol., vol. 8, pp. 1097-1106, July 1990.

Alberto Bononi received the "Laurea in Ingegneria Elettronica" from the University of Pisa, Pisa, Italy, in 1988. In 1989 he was a visiting researcher at the University of Parma, Parma, Italy, working on coheren optical communications. In 1990 he worked at GEC-Marconi Hirst Research Centre in Wembley, UK, on a Marconi S.p.A. Project on coherent FSK systems. He received the M.A. degree in electrical engineering from Princeton University, NJ, in 1992, where he is currently completing the Ph.D. program. His current research interests include system design and performance issues in fast packet switching and high-speed all-optical networks.

Paul R. Prucnal received the A.B. degree, summa cum laude, from Bowdoin College, Brunswick, ME in 1974, and the M.S., M. Phil. and Ph.D. degrees from Columbia University in 1976, 1978, and 1979, respectively. He was an Assistant Professor of Electrical Engineering at Columbia University from 1979 to 1984 , and he was an Associate Professor from 1984-1987. He was a member of the Executive Board of Columbia's NSF Engineering Research Center in Telecommunications Research from 1985-87. He is now a Full Professor at Princeton University, where he is Director of the Lightwave Communications Research Laboratory and recently served as Acting Director of the newly established New Jersey Advanced Technology Center in Photonics and Optoelectronic Materials. He has taught courses in the areas of fiber-optic communications systems, quantum electronics, and digital signal processing. He has been a Technical Consultant for Philips Labs, Optical Information Systems Inc., GTE Labs, IBM, Dove Electronics, and AT \& T Bell Labs. Professor Prucnal has published more than fifty journal papers in the areas of optical networks, photonic switching, optical techniques for advanced VLSI/VHSIC interconnections and optical signal processing, and holds three patents. He is a Fellow of the IEEE, and a member of OSA and SPIE, and he is an Associate Editor of the IEEE Transactions on Communications, the IEEE Circuits and Devices Magazine, and the IEEE Lightwave Communications Magazine.

Fabrizio Forghieri, photograph and biography not available at the time of publication. 